\title{
Impacted wire basket in the main pancreatic duct; How to Jailbreak
}

\section{Kumarasinghe KAPR* and Pathirana AA \\ Colombo South Teaching Hospital, Sri Lanka}

\section{Background}

Pancreatic calculi occur in about $50 \%$ of patients with chronic pancreatitis [1-3]. Of these, large intraductal calculi are seen in hereditary pancreatitis. These intraductal calculi causing duct obstruction results in intraductal hypertension, which would ultimately lead to chronic pain. Removal of intraductal stones (causing ductal obstruction), either endoscopically or surgically is recommended when patients with $\mathrm{CP}$ present with abdominal pain. While multiple calculi filling the ducts are treated surgically, when a few calculi are noted in the proximal duct, endscopic removal is the treatment of choice.

However, these stones are harder, compared to biliary calculi. Fragmentation using the mechanical lithotripter is not always possible. In such situations, ESWL or Laser lithotripsy might be helpful. Once the calculi are of a suitable size to trap in a wire basket, they could be removed through the papilla endoscopically.

\section{History}

A 42-year-old woman presented with upper abdominal pain related to meals suggestive of pancreatic origin, of 6 months duration. She had no exocrine or endocrine insufficiency. She underwent CECT abdomen and was found out to have pancreatic parenchymal and intra ductal calcifications, predominantly located in the head of the pancreas. Pancreatic duct was dilated to $5 \mathrm{~mm}$.

At an initial ERCP, PD stenting with a 7F $10 \mathrm{~cm}$ plastic stent had been placed in the MPD and she had defaulted for 8 months, as her symptoms had subsided.

She presented again with pain, not responding to simple analgesics. Imaging revealed a retained PD stent with ductal calculi. She underwent selective pancreatic cannulation and sphincteortomy. Previous stent was removed and pancreatography was done. It showed a dilated pancreatic duct with few intra ductal calculi (Figures 1 and 2).

Extraction with a wire basket (Olympus-Tetra Catch V-FGV432P) was attempted. Few smaller calculi were removed, however, while attempting to retrieve a larger stone, the wire basket got impacted within the PD, just distal to the ampulla. Attempts at releasing the basket failed. Then the wire of the basket was divided at the handle and the scope was removed. The wire of the basket was attached to the Mechanical lithotriptor (Olympus BML-11OA-1). While attempting to crush the wire of the baskets broke from the proximal end.

Available option was to take the patient to theatre, perform a duodenotomy and retrieve the stone and the basket after papillotomy.

However, as a last resort, it was decided to try ESWL with the basket in situ. For this, the patient was transferred to a hospital with ESWL facility, which was performed within 2 hours of the impaction.
Fortunately, this worked, and it was possible to remove the intact basket (Figure 3).

The patient was subjected to a repeat ERCP to exclude bleeding and duodenal injuries, and a $5 \mathrm{~F} 10 \mathrm{~cm}$ plastic stent was inserted to reduce the risk of post procedure pancreatitis. Her post procedure recovery was uneventful and was discharged on day two.

\section{Discussion}

Main objective of extraction of PD stones is to reduce the intraductal hypertension. There are several methods of treating PD stones. ERCP with or without ESWL is considered medical treatments. Surgically, pancreatic duct drainage procedures and resection procedures are performed. Three Randomized control trials have compared endoscopic treatment vs surgical drainage procedures, in that shortterm incidence of complications, pain relief and length of hospital stay is equal to surgical group. In long term surgical arm demonstrated a better symptom control and less re-intervention rate [4-6]. Although surgical options have been found to have better long-term symptom control, when compared with endoscopic options [4-6], most centres offer endoscopic approaches initially for those who are suitable for it (few stones, located proximally, without strictures)

Endoscopic balloon or basket retrieval is indicated in small, floating calculi which are $<5 \mathrm{~mm}$ in size. Adequate pancreatic sphincterotomy is helpful before extraction [7].

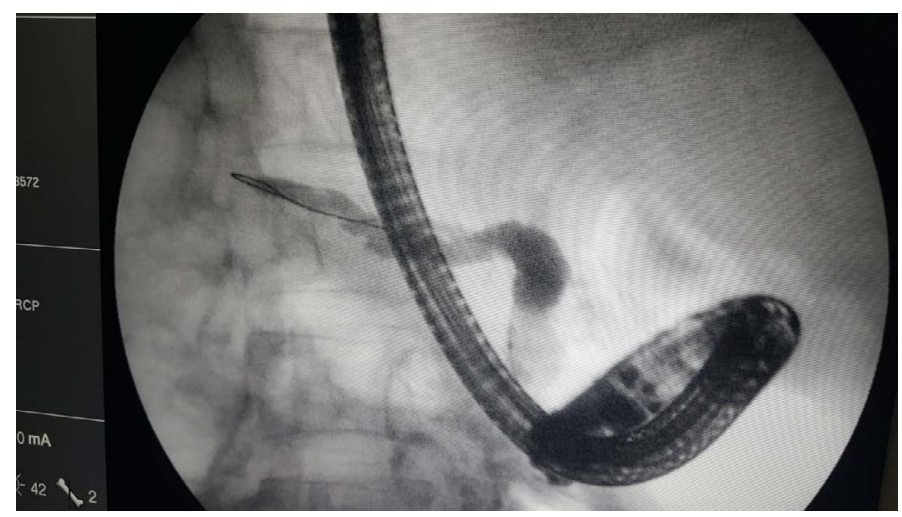

Figure 1. Pancreatogram showing dilated pancreatic duct with intraductal calculi Proceeded with a wire basket extraction of PD stones.

Correspondence to: Kumarasinghe KAPR, Colombo South Teaching Hospital, Sri Lanka, Tel: +94777914061; E-mail: prabath14@gmail.com

Received: December 03, 2017; Accepted: December 20, 2017; Published: December 23, 2017 


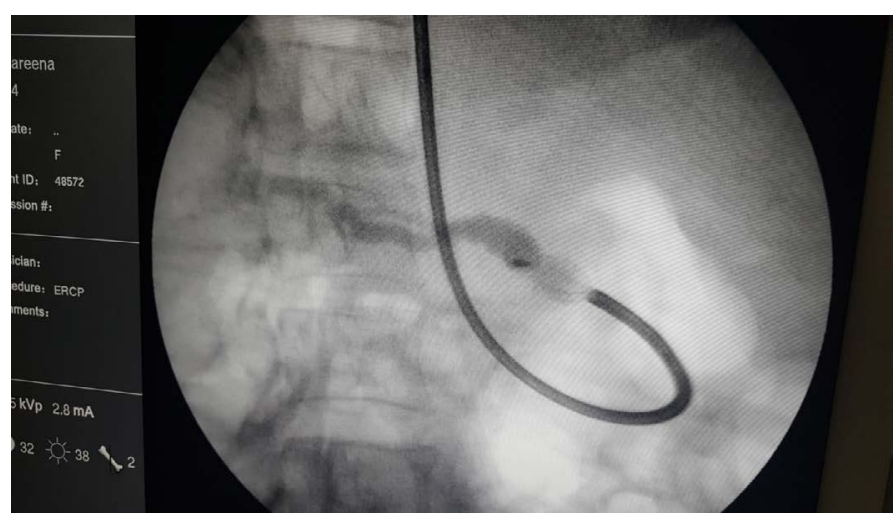

Figure 2. Impacted wire basket with a stone.

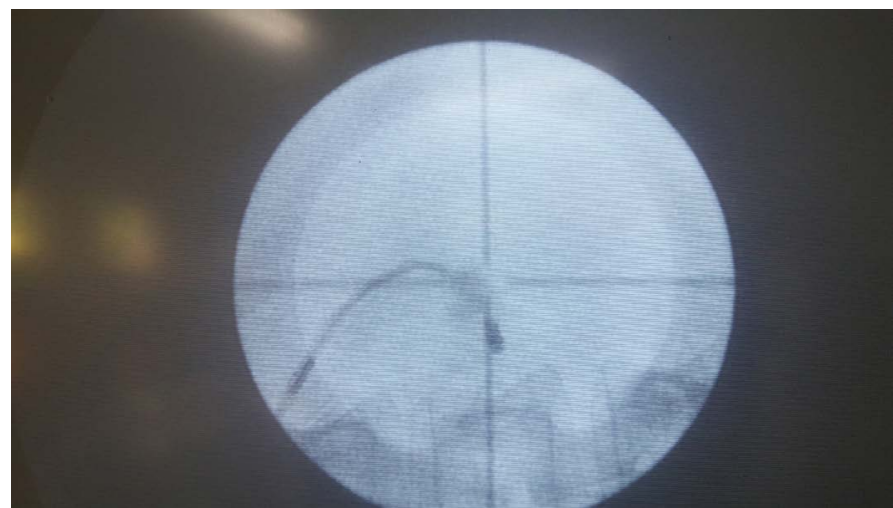

Figure 3. Fragmented calculus after ESWL.

According to The European Society of Gastrointestinal Endoscopy (ESGE) guidelines, MPD stones $>5 \mathrm{~mm}$ should undergo ESWL to fragment them before attempting ERCP, as ERCP alone reults in higher failure rates [8]. ESWL can be used in all uncomplicated PD stones. Main objective is to fragment stones in to tiny particles $<3 \mathrm{~mm}$, so they may be amenable to ERCP. ESWL also reduces the density of the stones [9]. A meta-analysis of Seventeen studies published between 1989 and 2002 demonstrated a stone clearance rate of ESWL ranging from $37 \%$ to $100 \%$ [10].

In another study of 125 patients, ESWL was found to enhances endoscopic interventions for PD stones when the mechanical lithotripsy fails [11]. ESWL has its own limitations. If the density of calculi $>820.5$ Hounsfield units on non -contrast computed tomography, there is a high failure rate. ESWL is not performed in the presence of extensive stone burden, and also its difficult to target an isolated stone in the tail of the pancreas without damaging the spleen. ESWL is contraindicated in pregnancy, abdominal aortic aneurysm, bleeding disorders and those with implanted cardiac pace makers. Complete stone clearance rate of this combined treatment is as high as $70 \%$. Newer endoscopic methods of treating MPD stones is using pancreatoscopy combined with laser or electrohydrolic lithotripsy.

\section{Conclusion}

Endoscopic PD stone retrieval is the method of choice for suitable cases of symptomatic MPD calculi. However, in the presence of large calculi $(>5 \mathrm{~mm})$ ESWL before endoscopic retrieval is recommended. If the basket gets impacted during retrieval, ESWL may be an option to try, before taking the patient to theatre for open exploration.

\section{References}

1. Tandan M, Talukdar R, Reddy DN (2016) Management of Pancreatic Calculi: An Update. Gut Liver 10: 873-880. [Crossref]

2. Rösch T, Daniel S, Scholz M, Huibregtse K, Smits M, et al. (2002) Endoscopic treatment of chronic pancreatitis: a multicenter study of 1000patients with long-term follow-up. Endoscopy 34: 765-771. [Crossref]

3. Inui K, Igarashi Y, Irisawa A, Ohara H, Tazuma S, et al. (2015) Japanese Clinical Guidelines for Endoscopic Treatment of Pancreatolithiasis. Pancreas 44: 1053-1064. [Crossref]

4. Dite P, Ruzicka M, Zboril V, Novotný I (2003) A prospective, randomized trial comparing endoscopic and surgical therapy for chronic pancreatitis. Endoscopy 35: 553-558. [Crossref]

5. Cahen DL, Gouma DJ, Laramée P, Nio Y, Rauws EA, et al. (2011) Long-term outcomes of endoscopic vs surgical drainage of the pancreatic duct in patients with chronic pancreatitis. Gastroenterology 141: 1690-1695. [Crossref]

6. Cahen DL, Gouma DJ, Nio Y, Rauws EA, Boermeester MA, et al. (2007) Endoscopic versus surgical drainage of the pancreatic duct in chronic pancreatitis. $N$ Engl J Med 356: 676-684. [Crossref]

7. Tandan M, Reddy DN, Santosh D, Vinod K, Ramchandani M, et al. (2010) Extracorporeal shock wave lithotripsy and endotherapy for pancreatic calculi-a large single center experience. Indian J Gastroenterol 29: 143-148. [Crossref]

8. Dumonceau JM, Delhaye M, Tringali A, Dominguez-Munoz JE, Poley JW, et al. (2012) Endoscopic treatment of chronic pancreatitis: European Society of Gastrointestinal Endoscopy (ESGE) Clinical Guideline. Endoscopy 44: 784-800. [Crossref]

9. Tandan M, Reddy DN (2011) Extracorporeal shock wave lithotripsy for pancreatic and large common bile duct stones. World J Gastroenterol 17: 4365-4371. [Crossref]

10. Guda NM, Partington S, Freeman ML (2005) Extracorporeal shock wave lithotripsy in the management of chronic calcific pancreatitis: a meta-analysis. JOP 6: 6-12. [Crossref]

11. Farnbacher MJ, Schoen C, Rabenstein T, Benninger J, Hahn EG, et al. (2002) Pancreatic duct stones in chronic pancreatitis: Criteria for treatment intensity and success. Gastrointest Endosc 56: 501-506. [Crossref]

Copyright: (C2017 Kumarasinghe KAPR. This is an open-access article distributed under the terms of the Creative Commons Attribution License, which permits unrestricted use, distribution, and reproduction in any medium, provided the original author and source are credited. 